\title{
Compassion fatigue, burnout, and compassion satisfaction in pediatric subspecialists during the SARS-CoV-2 pandemic
}

\author{
Samuel M. Kase ${ }^{1,5}$, Jeanie L. Gribben ${ }^{2,5}$, Katherine F. Guttmann ${ }^{3}$, Elisha D. Waldman ${ }^{4}$ and Andrea S. Weintraub ${ }^{3 凶}$ \\ (c) The Author(s), under exclusive licence to the International Pediatric Research Foundation, Inc 2021
}

BACKGROUND: The aim of this study was to explore factors contributing to compassion fatigue (CF), burnout (BO), and compassion satisfaction (CS) during the severe acute respiratory syndrome coronavirus-2 pandemic in pediatric subspecialists. METHODS: The Compassion Fatigue and Satisfaction Self-Test (CFST) and a questionnaire of personal/professional characteristics were distributed electronically to pediatric subspecialists.

RESULTS: There were no significant differences in pre- and early-pandemic CF, BO, and CS scores. Nearly $40 \%$ of respondents felt their contributions to the pandemic were not valued by their institutions. Higher CF scores were significantly associated with: higher BO score; "I have put myself at increased risk through my work"; working in one's specialty $>50 \%$ of time; distress about mental health and/or future uncertainty. Higher BO scores were significantly associated with: higher CF score; "Self-care is not a priority"; emotional depletion. Higher CS scores were significantly associated with: "My institution values my contribution to the COVID-19 crisis"; workplace debriefs; pet therapy.

CONCLUSIONS: The pandemic has only increased the need for physicians to receive social/emotional support from their institution and to feel their workplace contributions are valued. Successful pre-pandemic workplace interventions may not adequately support physicians during the pandemic. Further study is needed to identify supports that best counter the pandemic's unprecedented challenges.

Pediatric Research (2022) 91:143-148; https://doi.org/10.1038/s41390-021-01635-y

\section{IMPACT:}

- The sentiment "My institution has valued my contribution to the Covid-19 crisis" was the only significant factor associated with lower BO scores and was also associated with higher CS scores in pediatric subspecialists.

- This study is the first comparison of pre- and early-pandemic CF, BO, and CS scores in a national cohort of pediatric subspecialists.

- When considering interventions to promote CS and mitigate CF and BO for pediatric subspecialists during and after the pandemic, institutional leadership must offer wellness programming focused on social/emotional supports and prioritize a culture that explicitly recognizes and values every physician's contributions.

\section{INTRODUCTION}

The global pandemic of coronavirus disease 2019 (Covid-19) caused by the severe acute respiratory syndrome coronavirus- 2 (SARS-CoV-2) has forced physicians to rapidly adapt clinical practices and their personal lives to meet unprecedented professional demands. Physicians have been redeployed to provide clinical care outside their specialties, worked extra shifts and longer hours, and faced devastating outcomes among patients, colleagues, and loved ones. In addition, by virtue of their work environment, physicians are at high risk of acquiring SARSCoV-2 and transmitting it to patients, colleagues, and family members. The heightened personal and professional tolls of practicing medicine during the pandemic are well-documented. ${ }^{1-4}$ Healthcare workers globally are reporting increased levels of depressive symptoms, acute stress, burnout (BO), and exhaustion, with clinical demands and lack of control over practice cited as major contributors. ${ }^{3,5,6}$ At the same time, healthcare providers may also be experiencing shared pandemic stressors of socioeconomic inequalities, unemployment, limited support services, and childcare/schooling issues. ${ }^{4}$

Professional distress in physicians can manifest as compassion fatigue (CF) and/or BO. ${ }^{7-9}$ CF is defined as vicarious traumatic stress experienced by medical caregivers with chronic exposure to patient and family suffering. ${ }^{10} \mathrm{BO}$ is occupational distress characterized by emotional exhaustion and feelings of depersonalization and low personal achievement. ${ }^{11}$ The long-term corollaries of $\mathrm{CF}$ and $\mathrm{BO}$ in physicians, including stress in personal relationships, low morale, decreased productivity, decreased

\footnotetext{
'Department of Pediatrics, The Icahn School of Medicine at Mount Sinai, New York, NY, USA. ${ }^{2}$ Weill Department of Medicine, New York Presbyterian Hospital-Weill Cornell Medical Center, New York, NY, USA. ${ }^{3}$ Division of Newborn Medicine, Department of Pediatrics, Icahn School of Medicine at Mount Sinai, New York, NY, USA. ${ }^{4}$ Division of Palliative Care, Lurie Children's Hospital of Chicago, Chicago, IL, USA. ${ }^{5}$ These authors contributed equally: Samuel M. Kase, Jeanie L. Gribben. ${ }^{凶}$ email: andrea.weintraub@mssm.edu
} 
patient satisfaction, substance abuse, and suicide, are welldocumented and have only been exacerbated by the SARS-CoV2 pandemic. $^{8,12-18}$ Despite this, physicians have persevered, demonstrating courage, selflessness, and compassion. Compassion satisfaction (CS) is work-related fulfillment related to providing patient care and feeling supported and productive in professional endeavors. CS is often described as a protective factor against $\mathrm{CF}$ and $\mathrm{BO} .^{19}$

We have previously described $\mathrm{CF}, \mathrm{BO}$, and $\mathrm{CS}$ in national cohorts of neonatologists (NICU), palliative care physicians (PC), pediatric critical care physicians (CC), pediatric emergency medicine physicians $(\mathrm{EM})$, and pediatric hematology-oncology physicians (HO). ${ }^{20-24}$ In the current study, we explored CF, BO, and CS scores in these pediatric subspecialists during the early months of the pandemic, and compared them to pre-pandemic scores. We hypothesized that early-pandemic CF and BO scores would increase and CS scores would decrease when compared with pre-pandemic scores. We also hypothesized that geographic differences in $\mathrm{CF}, \mathrm{BO}$, and $\mathrm{CS}$ scores would emerge during the pandemic.

\section{METHODS}

The modified Compassion Fatigue and Satisfaction Self-Test (CFST), which contains 54 statements, with 18, 13, and 23 items on the $C F, B O$, and CS scales, respectively (Supplemental Table 1), was used to assess physician $\mathrm{CF}, \mathrm{BO}$, and $\mathrm{CS}^{20-24}$ Email addresses for physicians in selected pediatric subspecialties (compiled in our prior studies of $\mathrm{CF}, \mathrm{BO}$, and $\mathrm{CS}^{20-24}$ ) were utilized to contact potential subjects for the current study. A brief description of the study, with a hyperlink to the CFST and questionnaire of personal demographic information (Supplemental Material 2: sex, age, race, members of household, changes in living situation in the setting of the pandemic, pre-existing conditions that increase the risk of contracting Covid-19, current feelings of distress, and self-care activities) and professional details (Supplemental Table 2: pediatric subspecialty, setting and location of clinical practice, professional roles/responsibilities during the pandemic, available institutional support/programs for physician wellness), was distributed anonymously via SurveyMonkey ${ }^{\oplus}$ to potential study participants on June 9, 2020. Geographic regions were determined using workplace zip code and defined by US census designations. The invitation to participate was re-sent to nonresponders on June 23 and July 14. This project was designated as exempt human research by the Institutional Review Board at the Icahn School of Medicine at Mount Sinai.

Individual survey responses were downloaded, coded, and entered into SPSS Statistics, version 24 (IBM, Armonk, NY). Participants reflected on the sentiments "I have put myself at significant risk as part of my clinical work" and "My institution has valued my contribution to the Covid-19 crisis" using a 5 -point Likert scale $(0=$ strongly disagree, $1=$ disagree, $2=$ neutral, $3=$ agree, $4=$ strongly agree). Responses were dichotomized into "agree" (scale options 2, 3, or 4) or "disagree" (scale options 0 or 1). As in our prior work, subscale scores for CF, BO, and CS were summed. ${ }^{20-24}$ For each subscale, internal reliability was evaluated using Cronbach $a$, and normality was assessed with histogram analysis. Descriptive statistics were calculated for subscale score and questionnaire responses. The Pearson $r$ was used to examine correlations between subscales and to identify relationships between study variables.

Univariate analyses across subspecialties were performed using independent $t$ tests. One-way between-group analyses of variance were performed to explore the impact of subspecialty on $\mathrm{CF}, \mathrm{BO}$, and $\mathrm{CS}$ scores. Linear regression models for $\mathrm{CF}, \mathrm{BO}$, and $\mathrm{CS}$ scores as a function of subspecialty and other risk factors significant at $p<0.05$ in univariable analyses were constructed. For each phenomenon, standardized coefficients ( $\beta$ values), $t$ statistics, and adjusted $R^{2}$ were determined across models.

One-way between-group analyses of variance were performed to compare our previously published, pre-pandemic (2016-2019) CF, BO, and CS scores in physicians in selected subspecialties ${ }^{20-24}$ with earlypandemic scores collected in June and July 2020.

\section{RESULTS}

\section{Characteristics of the study population}

A total of 499 surveys were included in our analysis (26\% response rate) (Fig. 1). The majority of participants self-identified as white and female, and practiced at academic medical centers (Table 1). The distribution of participants across subspecialties was fairly equal, with the exception of PC. The majority of participants were from the Northeast and Southern United States. Nearly all participants reported feeling distressed about some aspect of

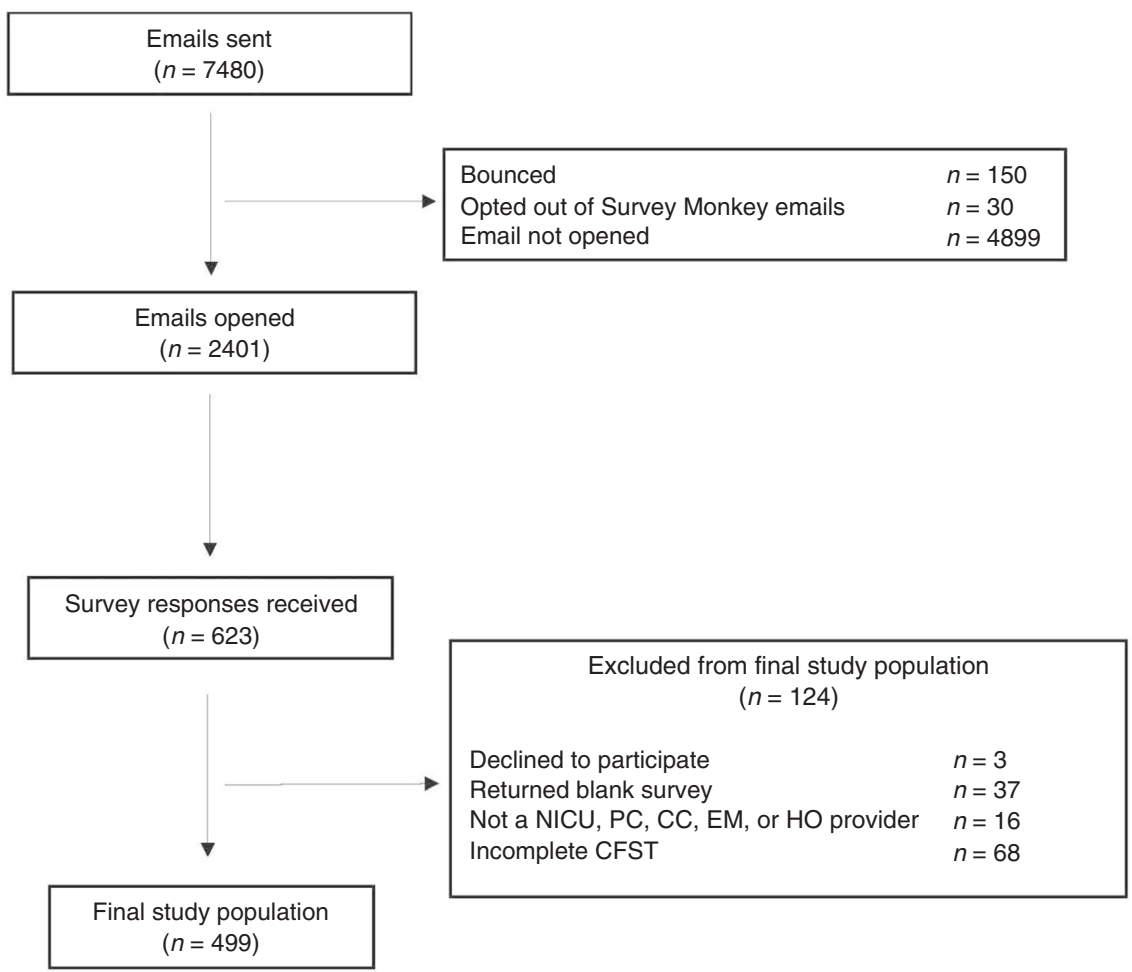

Fig. 1 Survey response and population. 
Table 1. Characteristics of the study population, June-July 2020 $(N=499)$.

\begin{tabular}{l} 
Gender, female \\
Age $(\mathrm{yr})(\mathrm{N}=48$ \\
$30-40$ \\
$41-50$ \\
$51-60$ \\
$\geq 61$ \\
Race $(\mathrm{N}=476)$ \\
\hline White \\
Black \\
Latinx \\
Asian \\
Other
\end{tabular}

US region where employed $(N=484)$

Northeast

Midwest

South

West

Pediatric subspecialty $(N=499)$

NICU

Palliative care

Pediatric critical care

Emergency medicine

Hematology-oncology

Pandemic effect on clinical practice environment $(N=498)$

Work in my own specialty $\geq 50 \%$ of the time

Redeployed outside of specialty

Work from home $\geq 50 \%$ of the time

$216(43.3)$

Furlough/unemployed

Pay cut

Working more frequently/longer hours

"My clinical work has placed me at increased risk" $(N=480)$

"My institution appreciates my contribution to the pandemic" $(N=477)$

Currently distressed about $(N=498)$

My health

My mental health

$166(33.3)$

Family health

Economic uncertainty

$287(57.6)$

$170(34.2)$

Working outside one's own specialty

Witnessing frequent patient suffering/death

Uncertain future

$46(9.2)$

Social isolation

Childcare/schooling

$329(66.1)$

$233(46.8)$

$146(29.3)$

Work-related issues

Current self-care activities $(N=497)$

Exercise

Outdoor activities

Engage in creative arts

$372(74.8)$

177 (35.6)

TV/Netflix

$345(69.4)$

Reading

\section{Table 1 continued}

Social media

Socialize with family/friends (in person or virtual)

Spiritual practice

187 (37.6)

Holistic practice

Mental health care

Engage in negative habits

Self-care is not a priority

their personal or professional lives on the day of the survey. When participants were asked to indicate which factors were causing them distress in the 3 months prior to the survey, the most frequently selected causes were concerns about "family health," "economic uncertainty," and an "uncertain future." When asked to reflect on the sentiment "I have put myself at significant risk as part of my clinical work," nearly $60 \%$ of participants affirmed this concern. When asked to evaluate the statement "My institution has valued my contribution to the Covid-19 crisis," $60 \%$ felt their efforts had been appreciated. Self-care activities of participants are listed in Table 1.

\section{Comparison of early- and pre-pandemic scores}

An overview of the pre- and early-pandemic CFST results is presented in Table 2. The previously published, pre-pandemic cohort was a combined population of 1876 pediatric subspecialists ( $\mathrm{NICU}=479, \mathrm{PC}=150, \mathrm{CC}=475, \mathrm{EM}=393, \mathrm{HO}=379$ ); $59 \%$ of this group was female and $79 \%$ was white. ${ }^{20-24}$ Cronbach $a$ values were $0.9,0.8$, and 0.9 for $\mathrm{CF}, \mathrm{BO}$, and $\mathrm{CS}$ scales, respectively, which suggested internally reliable scales. There were no significant differences in $\mathrm{CF}, \mathrm{BO}$, and $\mathrm{CS}$ scores across the subspecialties under investigation during the early months of the pandemic (Table 2). There were also no significant differences in pre- and early-pandemic CF, BO, and CS scores across these subspecialties. Prior to the pandemic, there were statistically significant differences in $\mathrm{CF}, \mathrm{BO}$, and $\mathrm{CS}$ scores across subspecialties (CF: $F(4,1871)=5.26, p<0.000$; BO: $F(4,1871)=3.33, p<$ 0.01 ; CS: $F(4,1871)=7.12, p<0.000)$, although the actual differences in mean scores between groups were small (effect size: $\eta^{2}=0.01$ for each scale) (Table 2). Post hoc comparisons indicated that prior to the pandemic, mean CF scores were significantly different between NICU and CC $(p<0.000)$ and between $\mathrm{CC}$ and $\mathrm{HO}(p<0.015)$, with the $\mathrm{CC}$ mean score higher in both instances. Mean BO scores were also significantly different between PC and CC $(p<0.006)$, with CC mean score higher than $P C$. Finally, mean CS scores were significantly different between NICU and CC $(p<0.001)$, PC and CC $(p<0.000)$, PC and EM $(p<$ $0.010)$, and $C C$ and $\mathrm{HO}(p<0.023)$, with $C C$ and EM mean scores lower in all instances. There were no significant differences in $C F$, $\mathrm{BO}$, or CS scores across geographic regions in the United States.

\section{Early-pandemic CF analyses}

Based on linear regression models, the following personal and professional factors were significant independent predictors of higher CF score: higher BO score; the sentiment "I have put myself at increased risk through my work"; working in one's own specialty at least $50 \%$ of the time; distress about "my mental health" and/or about an "uncertain future" (Table 3A). In contrast, a higher CS score was the sole significant independent predictor of a lower CF score.

\section{Early-pandemic BO analyses}

The following factors were each significant predictors of higher BO score: higher CF score; the sentiment "Self-care is not a priority for me"; and emotional depletion (Table 3B). In contrast, the sentiment "My institution has valued my contribution to the 
Table 2. Characteristics of the study instrument across pediatric subspecialties before SARS-Cov2 pandemic and in June-July 2020.

\begin{tabular}{|c|c|c|c|c|c|c|c|c|c|c|}
\hline & $\begin{array}{l}\text { Pre, } \\
N\end{array}$ & $\begin{array}{l}\text { Pre, mean } \pm \\
\text { SEM }\end{array}$ & $\begin{array}{l}\text { Pre, } \\
\text { median (IQR) }\end{array}$ & $\begin{array}{l}\text { Pre, } \\
\text { min }^{\mathrm{a}}\end{array}$ & $\begin{array}{l}\text { Pre, } \\
\text { max }^{b}\end{array}$ & $\begin{array}{l}\text { Early, } \\
N\end{array}$ & $\begin{array}{l}\text { Early, mean } \pm \\
\text { SEM }\end{array}$ & $\begin{array}{l}\text { Early, } \\
\text { median (IQR) }\end{array}$ & $\begin{array}{l}\text { Early, } \\
\text { min }^{\mathbf{a}}\end{array}$ & $\begin{array}{l}\text { Early, } \\
\text { max }^{b}\end{array}$ \\
\hline $\mathrm{NICU}$ & 479 & $16.9 \pm 0.5$ & $14(13)$ & 0 & 80 & 115 & $17.9 \pm 1.0$ & $16(14)$ & 0 & 43 \\
\hline PC & 150 & $18.0 \pm 0.8$ & $16(12)$ & 2 & 59 & 22 & $16.2 \pm 1.5$ & $15(12)$ & 8 & 30 \\
\hline CC & 475 & $20.1 \pm 0.5$ & $18(16)$ & 0 & 70 & 122 & $19.2 \pm 0.9$ & $17.5(12)$ & 0 & 56 \\
\hline EM & 390 & $19.0 \pm 0.6$ & $17(13)$ & 1 & 75 & 131 & $18.3 \pm 1.0$ & $16.1(12)$ & 0 & 76 \\
\hline \multicolumn{11}{|c|}{ Burnout subscale } \\
\hline $\mathrm{NICU}$ & 433 & $19.2 \pm 0.4$ & $17(11)$ & 2 & 52 & 114 & $19.5 \pm 0.7$ & $18(10)$ & 3 & 40 \\
\hline PC & 150 & $17 \pm 0.7$ & $17(11)$ & 1 & 41 & 20 & $20.9 \pm 1.7$ & $20(12)$ & 7 & 35 \\
\hline $\mathrm{CC}$ & 475 & $20.5 \pm 0.4$ & $19(12)$ & 2 & 49 & 122 & $21.2 \pm 0.9$ & $20(13)$ & 0 & 51 \\
\hline PC & 150 & $89.8 \pm 1.0$ & $91(19)$ & 53 & 115 & 20 & $85.3 \pm 3.1$ & 87 (19) & 55 & 105 \\
\hline $\mathrm{CC}$ & 475 & $83.8 \pm 0.7$ & 85 (19) & 42 & 115 & 122 & $83.5 \pm 1.3$ & 84.5 (19) & 38 & 114 \\
\hline EM & 390 & $85.0 \pm 0.8$ & $87(22)$ & 28 & 115 & 129 & $86.6 \pm 1.4$ & $89(22)$ & 48 & 114 \\
\hline $\mathrm{HO}$ & 363 & $86.9 \pm 0.7$ & $89(18)$ & 28 & 114 & 106 & $83 \pm 1.6$ & $84.5(23)$ & 39 & 115 \\
\hline
\end{tabular}

SEM standard error of the mean, IQR interquartile range, NICU neonatology, $P C$ palliative care, $C C$ critical care, EM emergency medicine, $H O$ hematologyoncology.

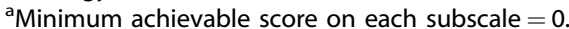

${ }^{\mathrm{b}}$ Maximum achievable score on Compassion fatigue subscale $=90$; Burnout subscale $=65$; Compassion satisfaction subscale $=115$.

Covid-19 crisis" was the only significant independent predictor of lower BO scores.

\section{Early-pandemic CS analyses}

The following factors were significant independent predictors of higher CS score: the sentiment "My institution has valued my contribution to the Covid-19 crisis," and availability of workplace debriefs and pet therapy (Table 3C). In contrast, higher BO score and distress about "my mental health" and "physical exhaustion" were significant independent predictors of lower CS scores.

\section{DISCUSSION}

We present the first comparison of pre- and early-pandemic data on $\mathrm{CF}, \mathrm{BO}$, and $\mathrm{CS}$ scores in a national cohort of pediatric subspecialists. Although we expected to find increases in CF and $\mathrm{BO}$ in the early-pandemic scores, no such differences were observed. There were some changes in scores when comparing subspecialists, although these did not follow patterns from which we can draw clear conclusions. For example, prior to the pandemic, CF scores in CC physicians were significantly higher than in NICU or HO. In the early pandemic, however, CF scores for CC physicians had decreased sufficiently to make the differences between these groups not statistically significant. One possible explanation is that the pandemic may have had an equalizer effect, such that universal causes of distress (e.g., concerns about an uncertain future, the health of family members, social isolation) diminished prior differences driven by subspecialty. Given these observations and difficult to distill trends, which likely mirror the complex and dynamic impact of pandemic-related stressors on physicians, personal and professional factors associated with an increase or decrease of $\mathrm{CF}, \mathrm{BO}$, and CS may help illuminate trends for further research.

\section{Factors impacting CF score}

Higher CF scores were associated with the sentiment, "I have put myself at increased risk through my work." The chronic stress of working at increased personal risk is both physically and emotionally exhausting. ${ }^{1,5}$ A sense of duty to care for patients and the related inability to extricate oneself from these risky situations may further contribute to CF. This lack of control or agency may also be reflected in the fact that distress regarding "uncertainty about the future" was significantly associated with a higher CF score. This is not surprising given the unclear duration of new norms created by the pandemic. ${ }^{6} \mathrm{~A}$ higher CS score was the only factor associated with lower CF scores, which suggests that CS is an important target for intervention.

\section{Factors impacting BO score}

Emotional depletion was significantly associated with a higher BO score, as was the sentiment "Self-care is not a priority for me." Chronic exposure to SARS-CoV-2, the uncertain course of the pandemic, and the resultant fatigue may drive such emotional exhaustion. ${ }^{2,25}$ Given the altered and unpredictable demands during the pandemic, participants may also have difficulty prioritizing self-care that might mitigate $\mathrm{BO}^{20,26,27}$ The sentiment "My institution has valued my contribution to the Covid-19 crisis" was the sole significant factor associated with lower BO scores. It was also associated with higher CS scores, and may provide an important point of intervention for institutions.

Prior to the pandemic, higher BO scores in our cohort of pediatric subspecialists were associated with distress about "administrative/ academic issues" and/or "coworkers". ${ }^{20-24}$ These associations were not seen in our current study during the early pandemic. This may be due to changes in academic pediatricians' work during the pandemic, with mutable schedules, work from home, and changing administrative demands potentially shifting sources of distress. 
Table 3. Significant predictors of compassion fatigue, burnout, and compassion satisfaction scores in pediatric subspecialists during the earlypandemic (linear regression models).

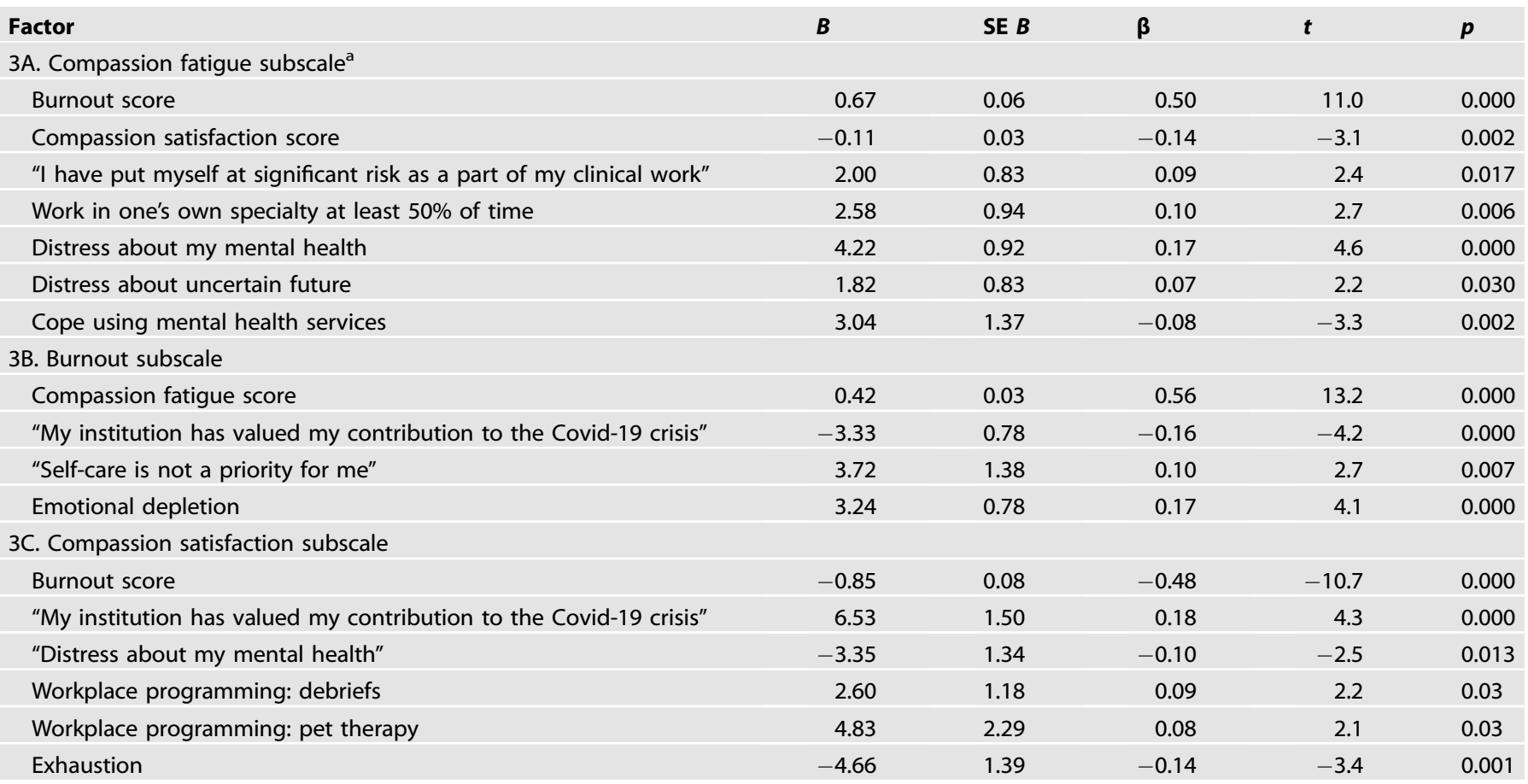

$B$ unstandardized coefficient, SE $B$ standard error of $B, \beta$ standardized coefficient, $t$ t-statistic.

${ }^{a}$ Covariates significant in univariate analyses included in the compassion fatigue regression modeling that did not reach statistical significance: female; working more/longer hours; distress about "own health," "family health," "economic issues," "witnessing frequent patient suffering/death," and/or "social isolation"; cope via "outdoor activities"; workplace support in the form of "peer-to-peer support"; "holistic programs" and/or "team building events"; feelings of "physical exhaustion" and/or "emotional depletion"; and the sentiment "My institution has valued my contribution to the Covid-19 crisis."

${ }^{\mathrm{b}}$ Covariates significant in univariate analyses included in the burnout regression modeling that did not reach statistical significance: female; working more/ longer hours; distress about "own health," "mental health," "family health," "economic issues," "social isolation," and/or "work-related issues"; cope via "mental health care"; workplace support in the form of "debriefs," "peer-to-peer support"; "holistic programs," "mental health programs," and/or "team building events"; feelings of "physical exhaustion"; and the sentiment "I have put myself at significant risk as part of my clinical work."

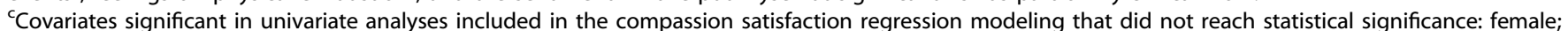
academic medical center; age; working more/longer hours; distress about "social isolation" and/or "child care"; cope via "outdoor activities," "mental health care," and/or "negative" habits; workplace support in the form of "peer-to-peer support"; "holistic programs," "mental health programs," and/or "team building events"; feelings of "emotional depletion"; and the sentiment "My institution has valued my contribution to the Covid-19 crisis."

\section{Factors impacting CS score}

As previously noted, the sentiment "My institution has valued my contribution to the Covid-19 crisis" was the sole significant factor associated with lower BO scores; notably, it was also significantly associated with higher CS scores. However, nearly $40 \%$ of participants expressed that they did not feel that their contribution was valued by their institution. This is an important finding for institutional leadership, as interventions aimed at making physicians feel valued can have a far-reaching impact. ${ }^{28}$ How institutional leadership expresses employee value and shows appreciation is not "one-size-fits-all" and requires meticulous attention. ${ }^{1,18}$ Prior to the pandemic, pediatric subspecialists reported wanting more social and emotional support from their institutions. ${ }^{28}$ That need has increased exponentially with the unpredictable demands of the pandemic. We need to understand how well-established, pre-pandemic institutional social/emotional supports translate to the virtual platform. ${ }^{26,29}$ If the essence of social/emotional support requires our physical presence together, how can we modify existing virtual platforms to provide what is needed? Finally, acknowledgement and exploration of positive emotions may galvanize joy and meaning in medicine and foster resilience. ${ }^{30}$ Our findings suggest potential targets for intervention to mitigate the negative impact of catastrophe and to amplify any potential positive outcomes.

\section{Limitations}

There are several limitations to our study. The survey response rate was relatively low, although it did fall within the range of response rates of our previous surveys on these phenomena, and for web-based surveys in subspecialist physicians. ${ }^{20-24,31}$ As with all survey studies, there is a risk of nonresponse bias. ${ }^{31}$ It is not possible to compare variables between those who responded to the survey and those who did not; it may well be that individuals at greatest risk for CF or BO did not participate in our studies, or alternatively, the reverse may be true. The generalizability of our findings may be limited by the fact that the majority of participants were white and female. Given the rapidly changing nature and protracted duration of the pandemic, our study represents one snapshot-albeit an important one-in time. Lastly, our findings are observed associations to which causality cannot be applied.

\section{CONCLUSION}

The personal and professional aftershocks of the current SARSCoV-2 pandemic for physicians will likely persist well into the foreseeable future. Our results suggest that institutions may be able to mitigate these effects using targeted interventions aimed at conveying to physicians that they are valued. When considering 
interventions to promote CS and mitigate $\mathrm{CF}$ and $\mathrm{BO}$ for pediatric subspecialists during and after the pandemic, a "one-size-fits-all" approach is inadequate. Institutions must prioritize an institutional culture that provides social/emotional supports and explicitly recognizes and values providers" contributions. Additional work is needed to develop and test interventions and to better understand their impact on $\mathrm{CF}, \mathrm{BO}$, and $\mathrm{CS}$.

\section{REFERENCES}

1. Ripp, J., Peccoralo, L. \& Charney, D. Attending to the emotional well-being of the health care workforce in a New York City Health System during the COVID-19 pandemic. Acad. Med. 95, 1136-1139 (2020).

2. Morgantini, L. A. et al. Factors contributing to healthcare professional burnout during the COVID-19 pandemic: a rapid turnaround global survey. PLOS ONE 15, e0238217 (2020).

3. Giusti, E. M. et al. The psychological impact of the COVID-19 outbreak on health professionals: a cross-sectional study. Front. Psychol. 11, 1684 (2020).

4. Blundell, R., Dias, M. C., Joyce, R. \& Xu, X. COVID-19 and inequalities. Fisc. Stud. 41, 291-319 (2020).

5. Lai, J. et al. Factors associated with mental health outcomes among health care workers exposed to coronavirus disease 2019. JAMA Netw. Open 3, e203976 (2020).

6. Shechter, A. et al. Psychological distress, coping behaviors, and preferences for support among New York healthcare workers during the COVID-19 pandemic. Gen. Hosp. Psychiatry 66, 1-8 (2020).

7. Meadors, P., Lamson, A., Swanson, M., White, M. \& Sira, N. Secondary traumatization in pediatric healthcare providers: compassion fatigue, burnout, and secondary traumatic stress. Omega 60, 103-1288 (2009).

8. van Mol, M. M., Kompanje, E. J., Benoit, D. D., Bakker, J. \& Nijkamp, M. D. The prevalence of compassion fatigue and burnout among healthcare professionals in intensive care units: a systematic review. PLoS ONE 10, e0136955 (2015).

9. Crowe, S., Sullivant, S., Miller-Smith, L. \& Lantos, J. D. Grief and burnout in the PICU. Pediatrics 139, e20164041 (2017).

10. Figley, C. R. In Compassion Fatigue (ed. Figley, C. R.) 1-20 (Taylor \& Francis, 1995).

11. Maslach, C. \& Jackson, S. E. The measurement of experienced burnout. J. Organ. Behav. 2, 99-113 (1981)

12. Meadors, P. \& Lamson, A. Compassion fatigue and secondary traumatization: provider self-care on intensive care units for children. J. Pediatr. Health Care 22, 24-34 (2008).

13. Sanchez-Reilly, S. et al. Caring for oneself to care for others: physicians and their self-care. J. Support. Oncol. 11, 75-81 (2013).

14. Williams, E. S., Rathert, C. \& Buttigieg, S. C. The personal and professional consequences of physician burnout: a systematic review of the literature. Med. Care Res. Rev. 19, 1077558719856787 (2019).

15. Restauri, N. \& Sheridan, A. D. Burnout and posttraumatic stress disorder in the coronavirus disease 2019 (COVID-19) pandemic: intersection, impact, and interventions. J. Am. Coll. Radiol. 17, 921-926 (2020).

16. Bradley, M. \& Chahar, P. Burnout of healthcare providers during COVID-19. Clevel. Clin. J. Med. https://doi.org/10.3949/ccjm.87a.ccc051 (2020).

17. Dzau, V. J., Kirch, D. \& Nasca, T. Preventing a parallel pandemic - a national strategy to protect clinicians' well-being. N. Engl. J. Med. 383, 513-515 (2020).

18. Shapiro, J. \& McDonald, T. B. Supporting clinicians during Covid-19 and beyond learning from past failures and envisioning new strategies. N. Engl. J. Med. 383, e142 (2020).

19. Stamm, B. H. In Treating Compassion Fatigue (ed. Figley, C. R.) 107-119 (Routledge, 2002).

20. Weintraub, A. S., Geithner, E. M. \& Waldman, E. D. Compassion fatigue, burnout, and compassion satisfaction in neonatologists in the US. J. Perinatol. 36 , 1021-1026 (2016)

21. Kase, S. M., Waldman, E. D. \& Weintraub, A. S. A cross-sectional pilot study of compassion fatigue, burnout, and compassion satisfaction in pediatric palliative care providers in the United States. Palliat. Support. Care 17, 269-275 (2019)
22. Gribben, J. L., Kase, S. M., Waldman, E. D. \& Weintraub, A. S. A cross-sectional analysis of compassion fatigue, burnout, and compassion satisfaction in pediatric critical care physicians in the United States. Pediatr. Crit. Care Med. 20, 213-222 (2019).

23. Gribben, J. L., MacLean, S. A., Pour, T., Waldman, E. D. \& Weintraub, A. S. A crosssectional analysis of compassion fatigue, burnout, and compassion satisfaction in pediatric emergency medicine physicians in the United States. Acad. Emerg. Med. 26, 732-743 (2019)

24. Weintraub, A. S., Sarosi, A., Goldberg, E. \& Waldman, E. D. A cross-sectional analysis of compassion fatigue, burnout, and compassion satisfaction in pediatric hematology-oncology physicians in the United States. J. Pediatr. Hematol. Oncol. 42, e50-e55 (2020).

25. Rodriguez, R. M. et al. Academic emergency medicine physicians' anxiety levels, stressors, and potential stress mitigation measures during the acceleration phase of the COVID-19 pandemic. Acad. Emerg. Med. 27, 700-707 (2020).

26. Mellins, C. A. et al. Supporting the well-being of health care providers during the COVID-19 pandemic: the CopeColumbia response. Gen. Hosp. Psychiatry 67, 62-69 (2020).

27. Rosa, W. E., Schlak, A. E. \& Rushton, C. H. A blueprint for leadership during COVID19. Nurs. Manag. 51, 28-34 (2020)

28. Kase, S. M., Gribben, J. L., Waldman, E. D. \& Weintraub, A. S. A pilot study exploring interventions for physician distress in pediatric subspecialists. Pediatr. Res. 88, 398-403 (2020).

29. Albott, C. S. et al. Battle buddies: rapid deployment of a psychological resilience intervention for health care workers during the COVID-19 pandemic. Anesth. Analg. 131, 43-54 (2020).

30. Rosenberg, A. R., Weaver, M. S., Fry, A. \& Wiener, L. Exploring the impact of the coronavirus pandemic on pediatric palliative care clinician personal and professional well-being: a qualitative analysis of U.S. Survey Data. J. Pain Symptom Manage. S0885-3924(20)30788-0 (2020).

31. Cunningham, C. T. et al. Exploring physician specialist response rates to webbased surveys. BMC Med. Res. Methodol. 15, 32 (2015).

\section{AUTHOR CONTRIBUTIONS}

S.M.K., J.L.G., K.F.G., E.D.W. and A.S.W. made substantial contributions to study conception and design; S.M.K. and J.L.G. to data acquisition; A.S.W. to data analysis; S.M.K., J.L.G. and A.S.W. to writing the first draft of the manuscript; K.F.G. and E.D.W. to critical revision of the manuscript for important intellectual content. All authors approved the final manuscript as submitted, and agree to be accountable for all aspects of the work.

\section{CONSENT STATEMENT}

Subject consent was not required for this study.

\section{COMPETING INTERESTS}

The authors declare no competing interests.

\section{ADDITIONAL INFORMATION}

Supplementary information The online version contains supplementary material available at https://doi.org/10.1038/s41390-021-01635-y.

Correspondence and requests for materials should be addressed to A.S.W.

Reprints and permission information is available at http://www.nature.com/ reprints

Publisher's note Springer Nature remains neutral with regard to jurisdictional claims in published maps and institutional affiliations. 